\title{
Quantitative Annular Dark-Field Imaging of Single-Layer Graphene
}

\author{
Shunsuke Yamashita ${ }^{1,2}$, Shogo Koshiya ${ }^{1}$, Kazuo Ishizuka ${ }^{1,3}$ and Koji Kimoto ${ }^{1,2}$ \\ 1. National Institute for Materials Science, 1-1 Namiki, Tsukuba, Ibaraki, Japan \\ 2. Department of Applied Chemistry, Kyushu University, 1-1 Namiki, Tsukuba, Ibaraki, Japan \\ 3. HREM Research Inc., 14-48 Matsukazedai, Higashimatsuyama, Saitama, Japan
}

Quantitative annular dark-filed (ADF) imaging in scanning transmission electron microscopy (STEM) has received much attention because it enables us to identify even the type and number of atoms of local structures. A quantification method of ADF imaging was proposed by LeBeau and Stemmer, in which the signal at each pixel is placed on an absolute scale by normalizing the current reaching an ADF detector by the incident probe current [1]. The method realized direct comparison between experimental and simulated ADF images without any arbitrary scaling parameters. They also reported nonlinear responses of the signal detection system of the microscope, thus the proposed method has a limitation that it can be used only on conditions that the signal detection system shows linear responses. In this study, we evaluated response properties of an ADF signal detection system and established a quantification procedure applicable to wider experimental conditions. Using the procedure, we acquired quantitative ADF images of single-layer graphene and compared with simulated images to investigate how accurately the scattering intensities match between experiments and simulations [2].

We used a $\operatorname{Titan}^{3}$ microscope (FEI) equipped with spherical aberration correctors (DCOR and CETCOR, CEOS) operating at an acceleration voltage of $80 \mathrm{kV}$. An ADF detector (Model 3000, Fischione) and an analog-to-digital (A/D) converter (DigiScan II, Gatan) were used. The response properties of the ADF signal detection system were evaluated by irradiating the ADF detector directly with the incident probe and investigating relationship between an input signal (incident probe current $I_{0}[\mathrm{pA}]$ ) and an output signal (ADF signal $S_{A D F}$ [count]). The incident probe current $I_{0}$ was measured in each experiment using a charge-coupled device (CCD) camera (UltraScan, Gatan), whose conversion efficiency was measured in advance. The response property depends on the contrast setting of the ADF detector, which corresponds to the voltage of a photo multiplier tube. The evaluation was conducted at various contrast settings between 50-100\%. After evaluating the response properties, curve fitting was carried out to obtain a conversion function which converts $S_{A D F}$ into current reaching the ADF detector, which is called ADF detector current $I_{A D F}[\mathrm{pA}]$. In the present quantification procedure, a quantitative contrast $Q_{A D F}[\%]$, i.e. $I_{A D F}$ normalized by $I_{0}$, was calculated from $S_{A D F}$ using both the conversion function and $I_{0}$. Acquired ADF images were converted into quantitative ADF images using the customized DigitalMicrograph (Gatan) scripts. Experimental conditions such as convergence semiangle of the probe and ADF detection angle range were precisely measured because they were important for quantitative comparison with simulated images. The STEM image simulation was performed using a multislice program (xHREM with STEM Extension, HREM). The simulation conditions were adjusted to the experimental conditions and quantitative contrast of the experimental ADF images were compared with that of simulated images. In STEM experiments, a commercial CVD graphene (TEM2000GL, ALLIANCE Biosystems) was used as a specimen.

The response properties at higher contrast settings showed nonlinearities as previously reported [1]. Thus, we obtained the conversion function that adequately reproduces experimental results by using a curve fitting. The conversion function enables us to convert $I_{A D F}$ from $S_{A D F}$ acquired under any value of 
the contrast settings between 50 and $100 \%$ because fitting parameters in the conversion function were also curve fitted as a function of the contrast setting. Figure 1 shows (a) a quantitative ADF image of graphene with 1-4 layers, (b) a high-resolution quantitative ADF image of single-layer graphene and (c) line profiles of quantitative contrast, respectively. The mean quantitative contrast, which was measured by averaging the value in areas including more than one unit cell, at a single-layer region was $0.054 \%$. The mean value of a simulated image was $0.053 \%$, thus the mean quantitative contrast exhibited excellent agreement between experimental and simulated images.

We have established a quantification procedure, in which the quantitative contrast is given as the ADF detector current normalized by the incident probe current. Since the quantification procedure fully implements the nonlinear responses for the first time, it allows us to acquire quantitative ADF images even under higher contrast settings, i.e. higher sensitivity conditions, which is indispensable to observation of nanomaterials. We applied the quantification procedure for observation of single-layer graphene and compared quantitative contrast between experiments and simulation. Consequently, it was revealed that the mean quantitative contrast of single-layer graphene showed good agreement. The quantitative ADF imaging could allow us to analyze atomic numbers of attached atoms on a graphene.

\section{References:}

[1] J M LeBeau and S Stemmer, Ultramicroscopy 108 (2008), p. 1653.

[2] S Yamashita et al, Microscopy (2015) in press (doi: 10.1093/jmicro/dfu115).

[3] This study was partly supported by the JST Research Acceleration Program and the Nano Platform Program of MEXT, Japan. The authors thank Dr. Nagai, Mr. Kurashima and Ms. Ohwada for support in the STEM experiments.
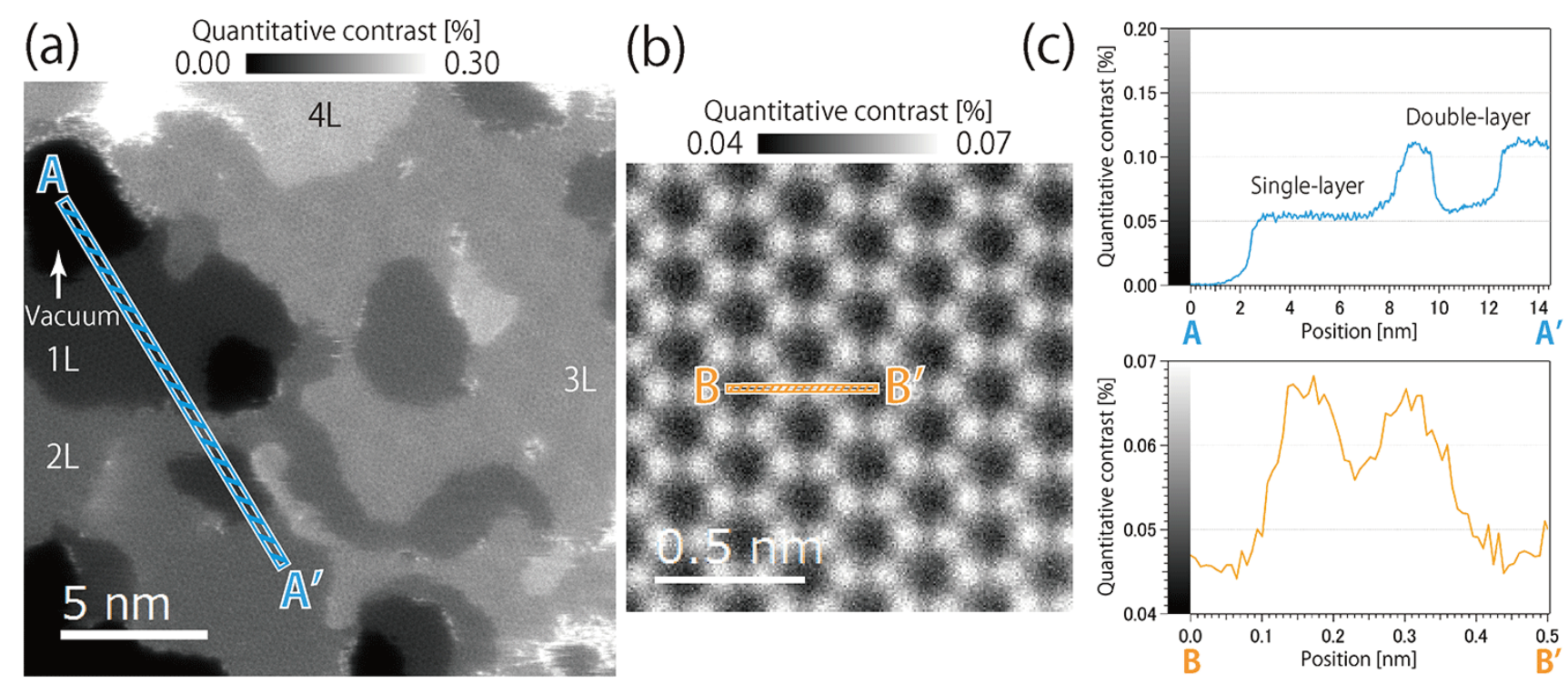

Figure 1. (a) Quantitative ADF image of graphene with 1-4 layers. (b) High-resolution quantitative ADF image of single-layer graphene. (c) Line profiles of quantitative contrast along A-A' in (a) and B$\mathrm{B}^{\prime}$ in (b), respectively. 